\title{
FOXA2 functions as a suppressor of tumor metastasis by inhibition of epithelial-to-mesenchymal transition in human lung cancers
}

\author{
Yunneng Tang ${ }^{1}$, Guangwen Shu ${ }^{1}$, Xinwang Yuan ${ }^{1}$, Naihe Jing ${ }^{1}$, Jianguo Song ${ }^{1}$ \\ ${ }^{1}$ Laboratory of Molecular Cell Biology, Institute of Biochemistry and Cell Biology, Shanghai Institutes for Biological Sciences, \\ Chinese Academy of Sciences, Shanghai 200031, China
}

The forkhead box transcription factor A2 (FOXA2) is an important regulator in animal development and body homeostasis. However, whether FOXA2 is involved in transforming growth factor $\beta 1$ (TGF- $\beta 1$ )-mediated epithelialto-mesenchymal transition (EMT) and tumor metastasis remains unknown. The present study showed that in human lung cancer cell lines, the abundance of FOXA2 positively correlates with epithelial phenotypes and negatively correlates with the mesenchymal phenotypes of cells, and TGF- $\beta 1$ treatment decreased FOXA2 protein level. Consistently, knockdown of FOXA2 promoted EMT and invasion of lung cancer cells, whereas overexpression of FOXA2 reduced the invasion and suppressed TGF- $\beta 1$-induced EMT. In addition, knockdown of FOXA2 induced slug expression, and ectopic expression of FOXA2 inhibited slug transcription. Furthermore, we identified that FOXA2 can bind to slug promoter through a conserved binding site, and that the DNA-binding region and transactivation region II of FOXA2 are required for repression of the slug promoter. These data demonstrate that FOXA2 functions as a suppressor of tumor metastasis by inhibition of EMT.

Keywords: FOXA2; EMT; TGF- $\beta 1$; lung tumors; slug

Cell Research (2011) 21: 316-326. doi:10.1038/cr.2010.126; published online 7 September 2010

\section{Introduction}

Tumor is known as one of the most dangerous threats to human health, and $\sim 90 \%$ of all cancer deaths arise from the metastatic spread of primary tumors. It has been widely recognized that epithelial-to-mesenchymal transition (EMT) is one of the major mechanisms for carcinoma progression, and the most compelling evidence for the involvement of EMT in carcinogenesis is the ability of multiple EMT regulators to enhance tumor formation and/or metastasis.

EMT is a process whereby epithelial cells lose their

Correspondence: Jianguo Song

Tel: +86-21-54921167

E-mail: jgsong@sibs.ac.cn

Abbreviations: FOXA2 (forkhead box transcription factor A2); TGF- $\beta$ (transforming growth factor $\beta$ ); EMT (epithelial-to-mesenchymal transition); ZO-1 (zonula occludens-1)

Received 14 May 2010; revised 8 July 2010; accepted 23 July 2010; published online 7 September 2010 epithelial cell features and acquire a mesenchymal morphological phenotype that is associated with some characteristic alterations at the molecular levels. EMT is also a highly fundamental process that is critical for embryonic development, wound healing, and fibrotic diseases [1-4]. Loss of cell junctions is considered as a crucial step in the progression of tumor invasion. Thus, loss of component molecules of adherens, gap, and/or tight junctions is the basic molecular event during EMT. One of the earliest events of EMT involves the disruption of tight junctions that connect epithelial cells, which is associated with a corresponding decrease or disappearance of tight junction proteins such as zonula occludens-1 (ZO-1) and occludin. Disruption of adherens junction complex, which contains E-cadherin and $\beta$-catenin, also contributes to the occurance of EMT [5].

EMT can be triggered by a variety of extracellular signals. Transforming growth factor $\beta$ (TGF- $\beta$ ), fibroblast growth factor, hepatocyte growth factor, epidermal growth factor family members, and insulin-like growth factor have all been shown to induce EMT in an auto- 
crine or paracrine manner [6]. In the past decade or so, it has been increasingly clear that TGF- $\beta$ is not only a potent inhibitor for the proliferation of epithelial cells but also, and more importantly, a potent inducer of EMT and apoptosis in many epithelial cells. The mechanism for TGF- $\beta$-induced EMT and apoptosis has, therefore, been a focus of study in recent years [7]. In hepatocyte cell lines and primary hepatocytes, TGF- $\beta$ has been shown to induce the concurrent apoptosis and EMT [8]. In A549 cells (human lung adenocarcinoma cells), TGF- $\beta$ cannot induce growth arrest and apoptosis, but induces a strong EMT response.

Forkhead box transcription factor A2 (FOXA2) belongs to the forkhead box (FOX) gene superfamily [9]. It is required for the formation of the node, notochord, nervous system, and endoderm-derived structures [10, 11]. Besides its importance in regulating embryo development, FOXA2 also plays a key role in metabolism.
It regulates normal bile acid homeostasis [12] and controls lipid homeostasis by affecting the clearance rate of fatty acids through oxidation and/or secretion of lipids in response to insulin [13, 14]. Although FOXA2 was found to be expressed in all types of neuroendocrine lung tumors [15], and was shown to be a key regulator in colorectal liver metastases [16], whether FOXA2 functions in human lung tumor development remains to be investigated.

In this study, we examined the expression levels of FOXA2 in several human lung cancer cell lines, and determined TGF- $\beta 1$-induced changes in the expression levels of FOXA2 in A549 cells. The correlations between the FOXA2 levels and the epithelial/mesenchymal phenotypes were analyzed. The results suggest that FOXA2 is a key target of TGF- $\beta 1$ in controlling EMT in human lung cancer cells.

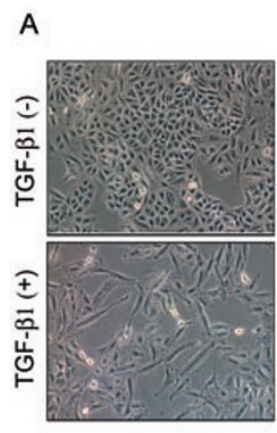

C

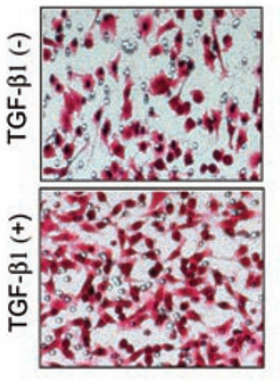

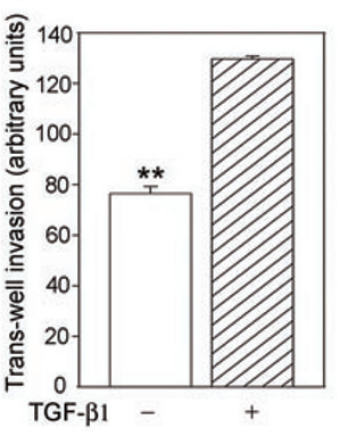

D
B

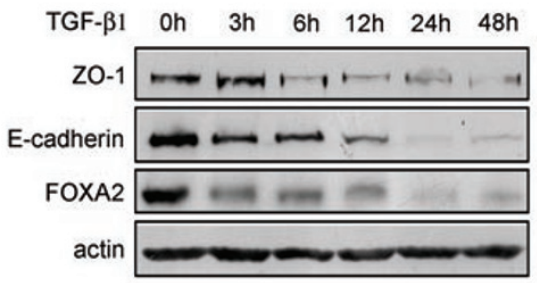

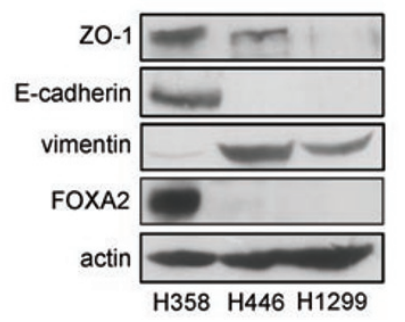
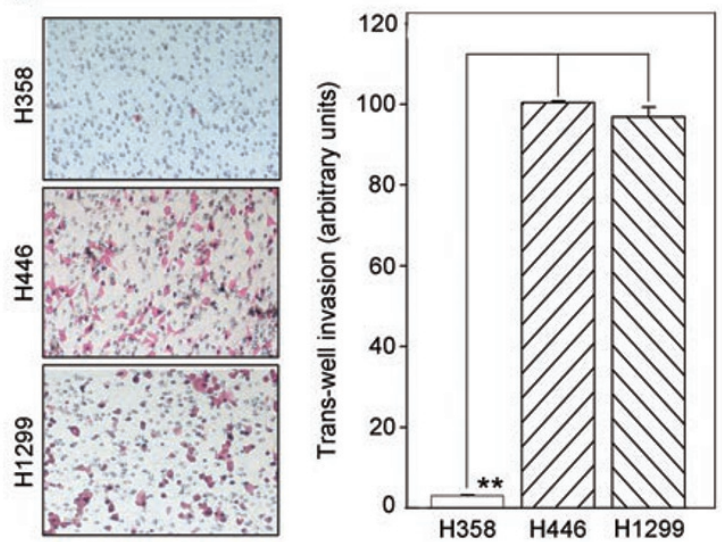

Figure 1 FOXA2 expression levels are related with EMT and increased migration ability of human lung cancer cells. (A) A549 cells were treated with TGF- $\beta 1(2 \mathrm{ng} / \mathrm{ml})$ for $48 \mathrm{~h}$. Cell morphological change of EMT was observed (left panel) and the invasiveness (middle and right) were detected by transwell assay (see Materials and Methods). (B) Levels of E-cadherin, ZO-1, and FOXA2 during EMT were examined by western blotting. (C) Expression levels of E-cadherin, ZO-1, vimentin, and FOXA2 in H358, H446, and H1299. (D) Transwell invasion results of H358, H446, and H1299 cells. The statistic data were shown on the right. 


\section{Results}

FOXA2 expression is positively correlated with epithelial identities and negatively correlated with mesenchymal identities in human lung cancer cells

TGF- $\beta$ is one of the most important regulators in EMT. It induces EMT in human lung cancer cells [17] and increases lung cancer cell migration ability [18]. A549 cells undergo EMT in response to TGF- $\beta 1$ treatment, as determined by cell morphology (Figure 1A, left) and increased cell migration ability (Figure 1A, middle and right). Decrease in ZO-1 and E-cadherin protein levels was also used to determine the occurrence of EMT, and interestingly, we found a fundamental decrease in FOXA2 protein levels, which is relatively an early event induced by TGF- $\beta 1$ (Figure 1B). To look into the potential linkage between FOXA2 and EMT, FOXA2 and EMT marker protein levels were examined in three other lung cancer cell lines. As shown in Figure 1C, in the H358 cell line, high FOXA2 levels are associated with high E-cadherin/ZO-1 and low vimentin levels, whereas low FOXA2 levels are associated with low E-cadherin/ ZO-1 and high vimentin levels in H446 and H1299 cell lines. Consistently, the transwell invasion abilities of H446 and H1299 are significantly stronger than that of H358 (Figure 1D). These results suggest that FOXA2

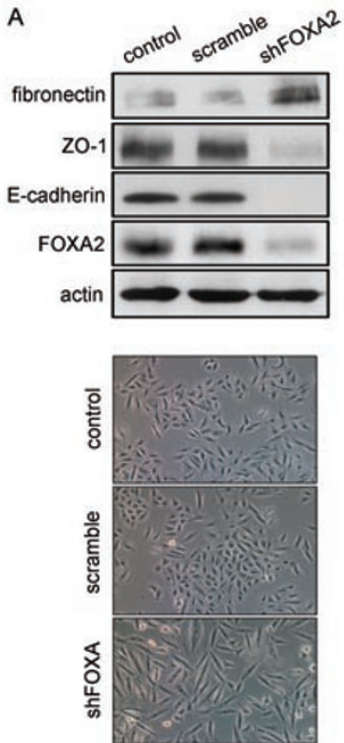

C

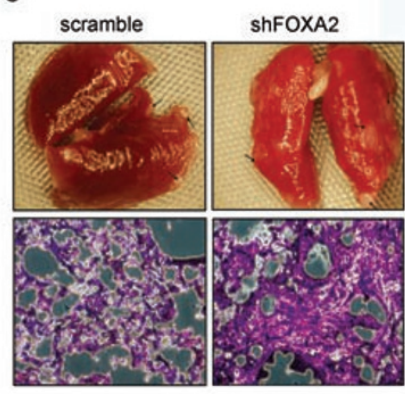

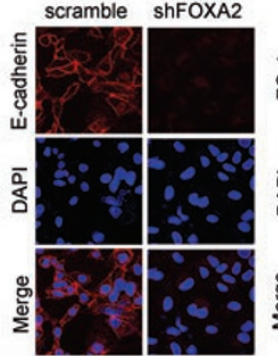
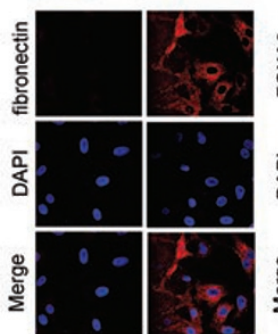

B

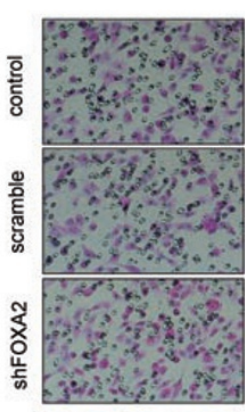

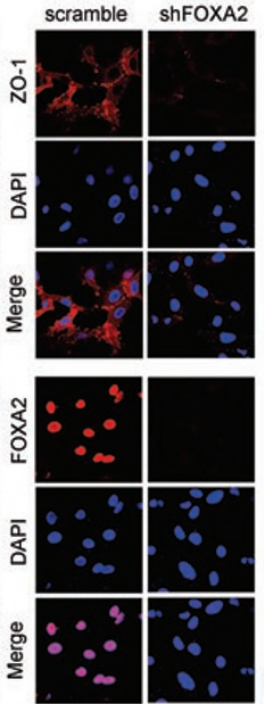

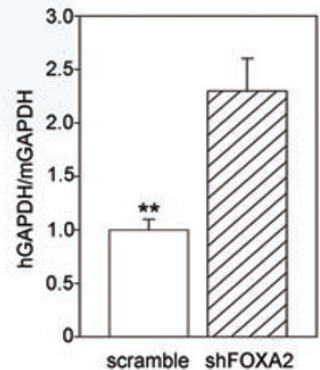

D

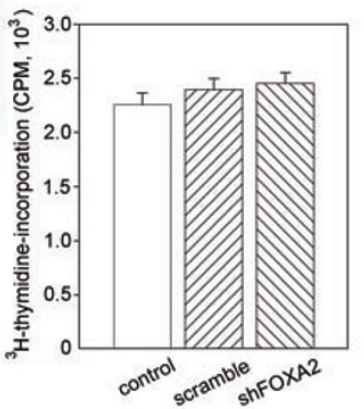

Figure 2 FOXA2 knockdown induces EMT and promote cell migration of A549 cells. (A) The effect of FOXA2 knockdown on EMT was examined by observing the morphology (left bottom) and measuring the expression levels of ZO-1, E-cadherin, and fibronectin by western blotting (left top) and immunofluorescence staining (middle and right). (B) Increased cell migration caused by knockdown of FOXA2 was determined by transwell assay. A reprentative result of three independent (left) and statistic data were presented. (C) At 8 weeks after tail vein injection of sramble or shFOXA2-transfected A549 cells, metastasis and/or tumor formation in lung was determined under a dissection microscope (left top) and by histological analysis using $\mathrm{H}$ and $\mathrm{E}$ (hematoxylin and eosin) staining (left bottom). Right panel, the relative concentration of circulating tumor cells in blood was determined by qRT-PCR. (D) ${ }^{3} \mathrm{H}$-thymidine incorporation analysis was performed to determine the effect of FOXA2 knockdown on the proliferation rate of cells. 
levels correlate with epithelial cell markers, and implicate FOXA2 in the control of EMT.

Knockdown of FOXA2 induces EMT and promotes invasion in human lung cancer cells

As there is no previous report showing the role of FOXA2 in EMT, it is important to determine whether FOXA2 functions in TGF- $\beta 1$-induced EMT in human lung cancer cells. We knocked down FOXA2 in A549 cells by using the lentivirus system. FOXA2 downregulation induced EMT, as shown by the increase in fibronectin level and decrease in ZO-1 and E-cadherin levels (examined by western blotting, Figure 2A, left top, and immunofluorescence staining, Figure 2A, middle and right). Morphological change from square-like epithelial to spindle-like mesenchymal phenotype of cells was also observed (Figure 2A, left bottom). FOXA2 downregulation also significantly increased the invasion ability of A549 cells (Figure 2B), as shown under a microscope (left) and by the statistic data (right). FOXA2 downregulation in the H358 human lung cancer cell line also induced EMT, as determined by decreased ZO-1 expression and increased vimentin expression (Supplementary information, Figure S1). These results indicate that FOXA2 is required for maintaining the epithelial phenotype and its downregulation can induce EMT and promote the invasiveness of human lung cancer cells.

The role of FOXA2 in controlling EMT suggests a role in tumor invasion and metastasis. We thus investigated the in vivo effect of FOXA2 on tumor invasion and metastasis. Knockdown of FOXA2 significantly increased the size of metastatic nodules of A549 cells in lung compared with scramble control cells, as detected under a dissection microscope (Figure 2C, left top). $\mathrm{H}$ and $\mathrm{E}$ (hematoxylin and eosin) staining (Figure 2C, left bottom) also showed that, 8 weeks after injection of FOXA2 RNAi A549 cells, significantly more space in pulmonary alveolus was occupied by tumor cells compared with the controls. Increased numbers of metastatic nodes in lung after tail vein injection was quantified as shown in Supplementary information, Figure S2A. The results further confirm that knockdown of FOXA2 in tu- mor cells promoted the formation of metastatic nodules in lung. FOXA2 knockdown also notably increased the number of circulating tumor cells, as detected by realtime PCR (Figure 2C, right). However, no obvious effect of FOXA2 knockdown on the proliferation rate of A549 cells was observed (Figure 2D), suggesting that FOXA2 knockdown increased invasion/metastasis of A549 cells through induction of EMT.

Ectopic expression of FOXA2 suppresses invasion and TGF- $\beta 1$-induced EMT

As FOXA2 knockdown induces EMT and increases invasion of A549 cells, FOXA2 overexpression might have an opposite effect. An N-terminal flag-tagged mouse FOXA2 or empty vector was transfected into A549 cells by using a lentivirus system. Overexpression of FOXA2 suppressed the TGF- $\beta 1$-induced EMT, as shown by inhibition of the increase of fibronectin and the decrease of E-cadherin (detected by western blotting, Figure 3A, left top, and immunofluorescence staining, Figure 3A, right), the cell morphological changes (Figure $3 \mathrm{~A}$, left bottom), and the invasion ability of A549 cells (Figure 3B). To test whether FOXA2 functions in other human lung cancer cell lines, FOXA2 was overexpressed in H446 and H1299 cells. Overexpression of FOXA2 in these cells also inhibited the EMT, as determined by the upregulation of ZO-1 and downregulation of fibronectin and $\mathrm{N}$-cadherin (Figure 3C, left), and suppression of the cell migration/invasion ability (Figure $3 \mathrm{C}$, middle and right). In contrast with the FOXA2 knockdown, overexpression of FOXA2 inhibited the ability of A549 cells to form metastatic nodules in lungs, as examined by a dissection microscope (Figure 3D, left top), quantification of the nodules (Supplementary information, Figure S2B), and by histological analysis (Figure 3D, left bottom). FOXA2 overexpression decreased the number of circulating tumor cells as well (Figure 3D, middle). ${ }^{3} \mathrm{H}$-thymidine incorporation analysis showed that overexpression of FOXA2 did not significantly affect the proliferation rate of lung tumor cells (Figure 3D, right), suggesting that the suppression of tumor invasion is not due to decreased cell proliferation rate.

Figure 3 Overexpression of FOXA2 suppresses EMT and invasion of human lung cancer cells. (A) Ectopic expression of flag-tagged FOXA2 in A549 cells suppress TGF- $\beta 1$ ( $1 \mathrm{ng} / \mathrm{ml} ; 2$ days)-induced EMT, as determined by the expression levels of fibronectin and E-cadherin detected by western blotting (left top) and immunofluorescence staining (right), and the cell morphological changes (left bottom). (B) Ectopic expression of FOXA2 decreases the invasiveness of A549 cells, as determined by transwell assay. Left, a representive result of three independent experiments; right, statistic data. (C) Changes in the expression levels of ZO-1, E-cadherin, and vimentin in H446 and H1299 cells overexpressing FOXA2 (left). Middle and right panels show decreased invasiveness of H1299 and H446 cells, as determined by transwell assay. (D) Mouse lungs (left top), $\mathrm{H}$ and $\mathrm{E}$ staining (left bottom), relative concentration of circulating tumor cells in blood (middle), and the ${ }^{3} \mathrm{H}$-thymidine incorporation analysis (right). 

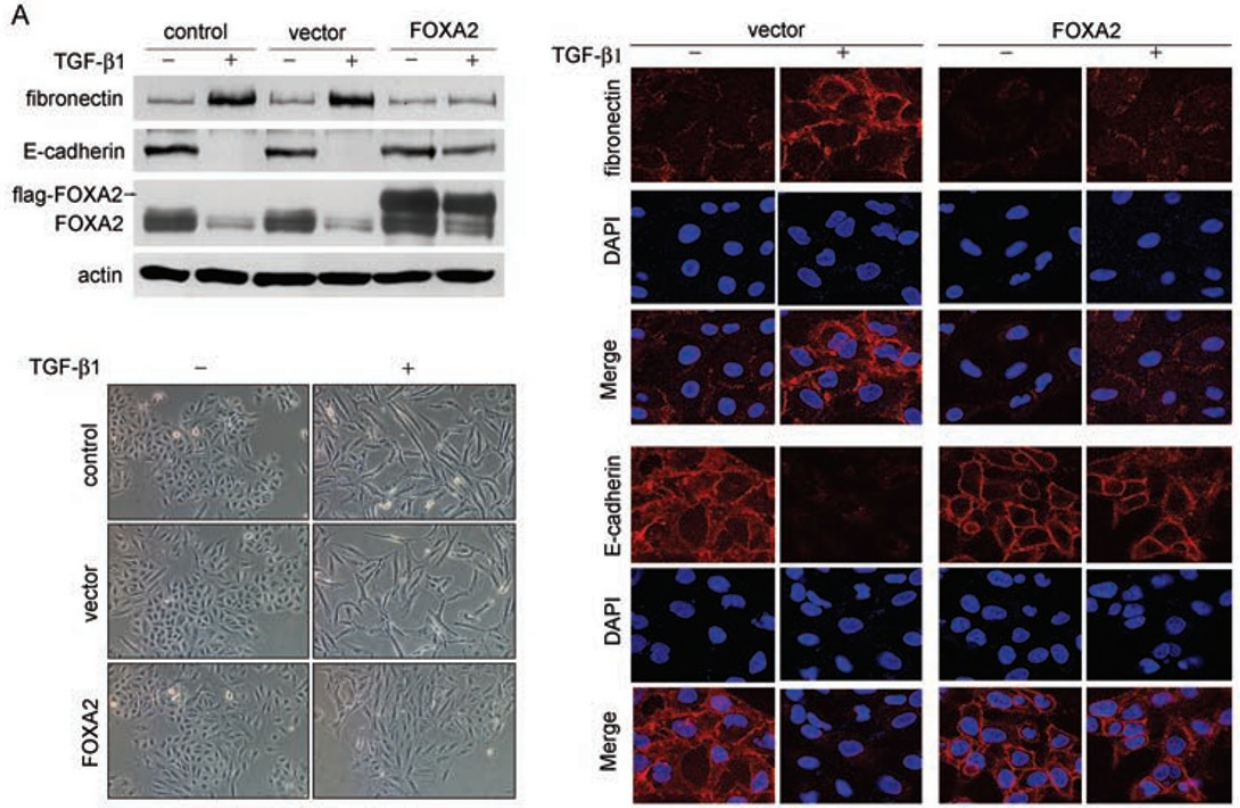

B TGF- $\beta 1$
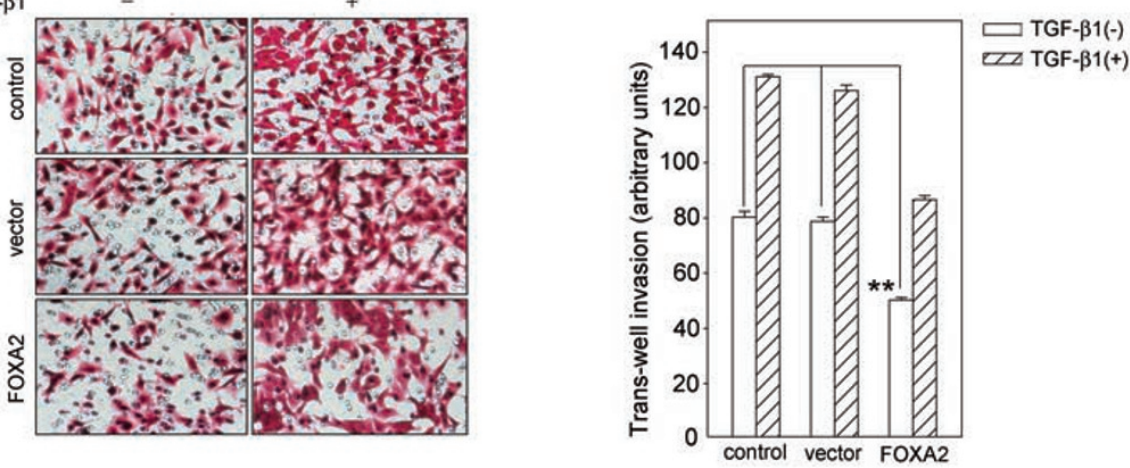

C
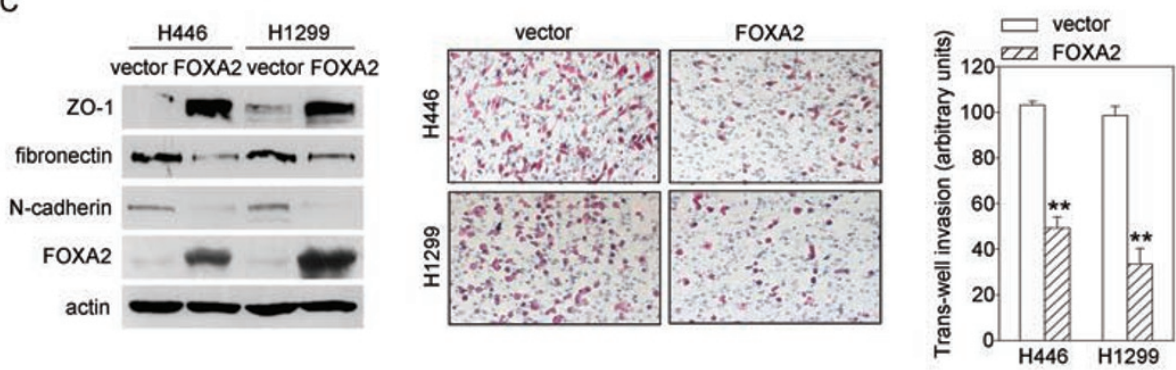

D
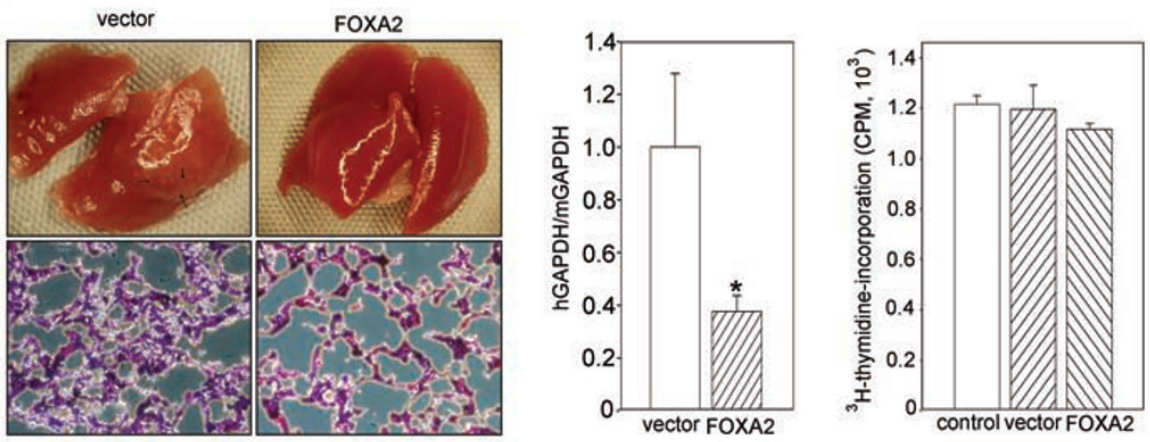

Cell Research | Vol 21 No 2 | Februray 2011 
FOXA2 inhibits slug expression in human lung cancer cells

In identifying the potential downstream molecules regulated by FOXA2, we observed that FOXA2 knockdown strongly increased the expression of slug, slightly increased the expression levels of snail and twist, but no sip 1 expression was detected (Figure 4A, left). Increased slug expression was also determined by western blotting (Figure 4A, right). As a member of the snail family of $\mathrm{C} 2 \mathrm{H} 2$-type zinc finger transcription factors [19], slug is a negative regulator of E-cadherin expression [20], and is critical for TGF- $\beta 1$-induced EMT $[21,22]$. Slug expression was shown to be an independent prognostic parameter for poor survival in colorectal carcinoma patients [23]. Slug is an important factor in regulating EMT in human lung cancer cells. Overexpression of slug in H358 cells (a human lung cancer cell line with strong epithelial identities) induced EMT, as shown by the decreased E-cadherin and ZO-1 levels and increased vimentin expression (Figure 4B). The results confirmed that slug plays a key role in EMT induction in human lung cancer cells. FOXA2 overexpression significantly inhibited the transcriptional activity of the slug promoter (Figure 4C). FOXA2 regulates the transcription of a variety of genes through a conserved binding site $[24,25]$. By performing blast analysis, two conserved putative FOXA2-binding sites (named as PBS1 and PBS2) in the slug promoter were detected (Figure 4D). In mice, PBS1 and 2 locate in -1351 to -1340 and -927 to -916 , and in humans, they locate in -1373 to -1362 and -1117 to -1106 , respectively. Thus, it is likely that FOXA2 may regulate slug promoter activity through one or both of these sites.

FOXA2 binds to slug promoter and suppresses slug transcription

To test the potential mechanism through which FOXA2 inhibits slug expression, we performed chromatin immunoprecipitation assay (ChIP assay) using two distinct pairs of primers: P1, detecting only the putative FOXA2-binding site 1; and P2, detecting only the putative FOXA2-binding site 2. As shown in Figure 5A, FOXA2 binds to the slug promoter through PBS1 but not PBS2. TGF- $\beta 1$ treatment ( $2 \mathrm{ng} / \mathrm{ml}, 2$ days), which decreased FOXA2 protein level, abolished the interaction. By electrophoretic mobility shift assay (EMSA) using a $5^{\prime}$ end biotin-labeled probe that contains triple repeat of PBS1, the binding between FOXA2 and PBS1 was verified (Figure 5B).

The FOXA2 protein contains four transactivation regions and a DNA-binding region [26, 27]. By consecu-
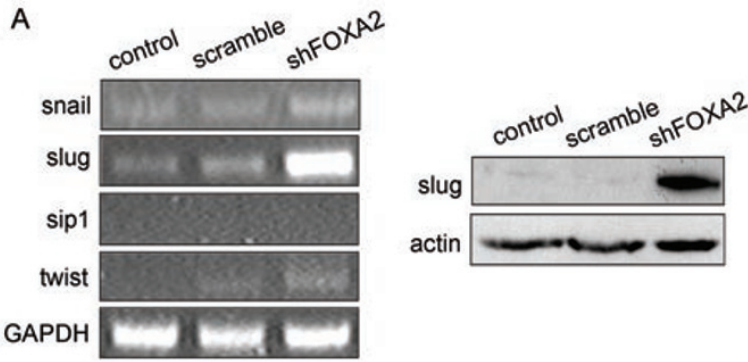

C

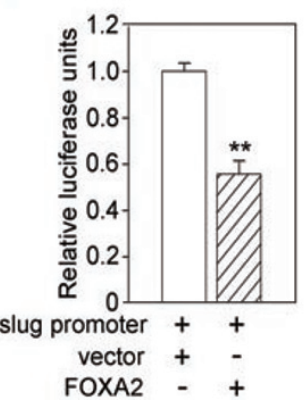

B

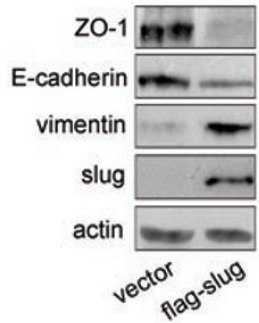

D

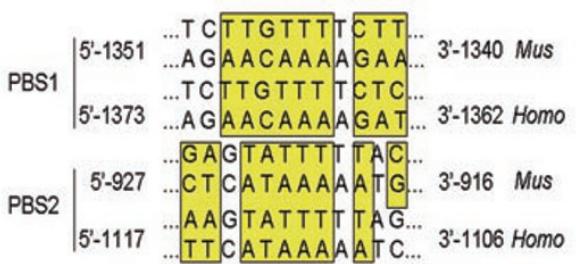

Figure 4 FOXA2 inactivates slug transcription in human lung cancer cells. (A) The expression of snail, slug, sip1, and twist in FOXA2 knockdown cells were analyzed by RT-PCR (left) and western blotting for slug (right). (B) EMT was examined by western blotting of ZO-1, E-cadherin, and vimentin in slug-overexprssed H358 cells. (C) Slug and FOXA2 were cotransfected into A549 cells and luciferase assay of the slug promoter activity was determined. (D) Blast analysis shows that there are two conserved putative FOXA2-binding sites (PBS1 and PBS2) in a slug promoter. 

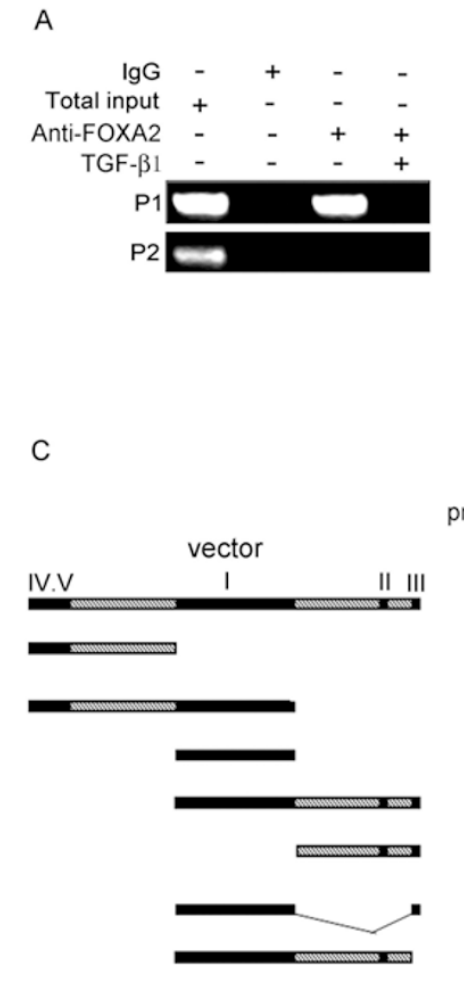
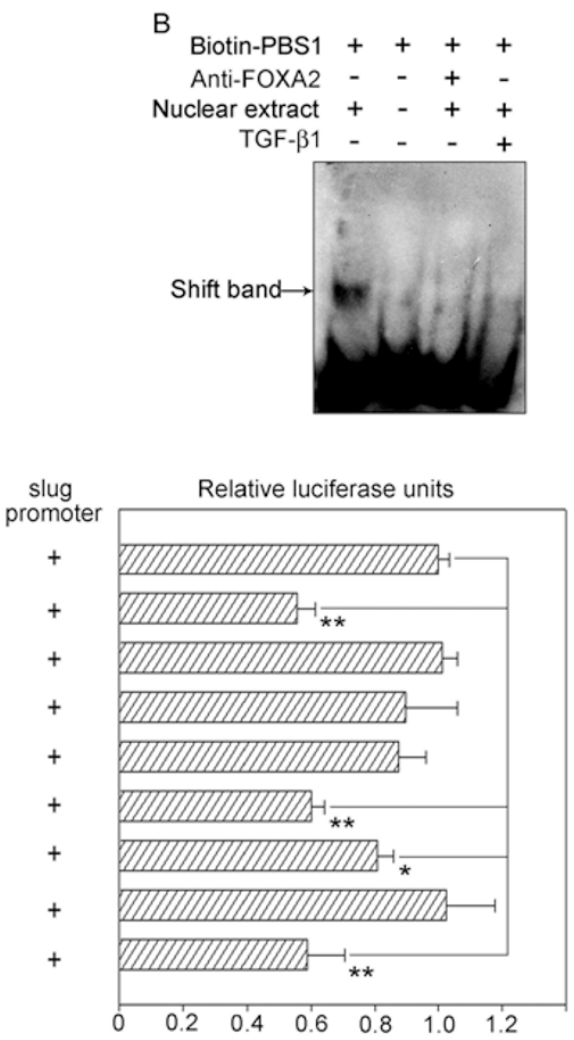

Figure 5 FOXA2 binds to PBS1 in slug promoter and suppress slug transcription through its transactivation region II. (A) ChIP assay was performed as described in Materials and Methods. The results show that FOXA2 binds to slug promoter through PBS1. (B) EMSA assay suggests that PBS1 mediates the binding between FOXA2 and slug promoter. (C) A summary of relative transcriptional activities produced by cotransfection of various FOXA2 deletions with slug promoter. Schematically shown are wild-type and deleted FOXA2 proteins, as well as conserved sequences that include: region I (winged, helix DNA-binding region), and regions II, III, IV, and V (transactivation regions). The caret indicates an internal deletion of the C-terminus (259/427). The bar graphs show the relative slug promoter's transcription activity.

tive DNA sequence truncations and a luciferase activity assay, we observed that both regions I (aa 157-258) and II (aa 372-385) of FOXA2 are required for the inhibition of slug promoter activity. By contrast, regions III (aa 445-458), IV, and V (aa 1-93) are not required for this inhibitory effect. The DNA-binding region (region I) plus the C-terminal transactivation region III showed no significant inhibition on the slug promoter. However, the DNA-binding region plus the C-terminal transactivation region II inhibited slug promoter's transcription activity (Figure 5C). These results indicate that upon binding to the slug promoter, FOXA2 inhibits slug transcription through its transactivation region II.

\section{Schematic illustration}

A schematic model was drawn to summarize our findings (Figure 6). In resting state of cells, FOXA2 binds to PBS1 in the slug promoter through its DNA-binding region, and suppresses slug transcription through its trans- activation region II. TGF- $\beta 1$ treatment decreases FOXA2 expression, thus activating slug transcription and leading to EMT in human lung cancer cells

\section{Discussion}

Lung cancer is one of the leading causes of cancerrelated deaths, with an incidence of 1.2 million per year and mortality of 1.1 million per year [28]. Its major etiological factor is tobacco smoking, which leads to inhalation of many carcinogens that play a role in causing the genetic and epigenetic changes in the respiratory epithelium during the multistage process of carcinogenesis [29]. Understanding the mechanisms involved in lung cancer invasion and metastasis is critical for lung cancer therapy. The genetic changes include activation of protooncogenes and inactivation of tumor suppressors. FOXA2 is a 'pioneer' transcription factor; it plays an indispensable role in embryo development, neural patterning, and or- 


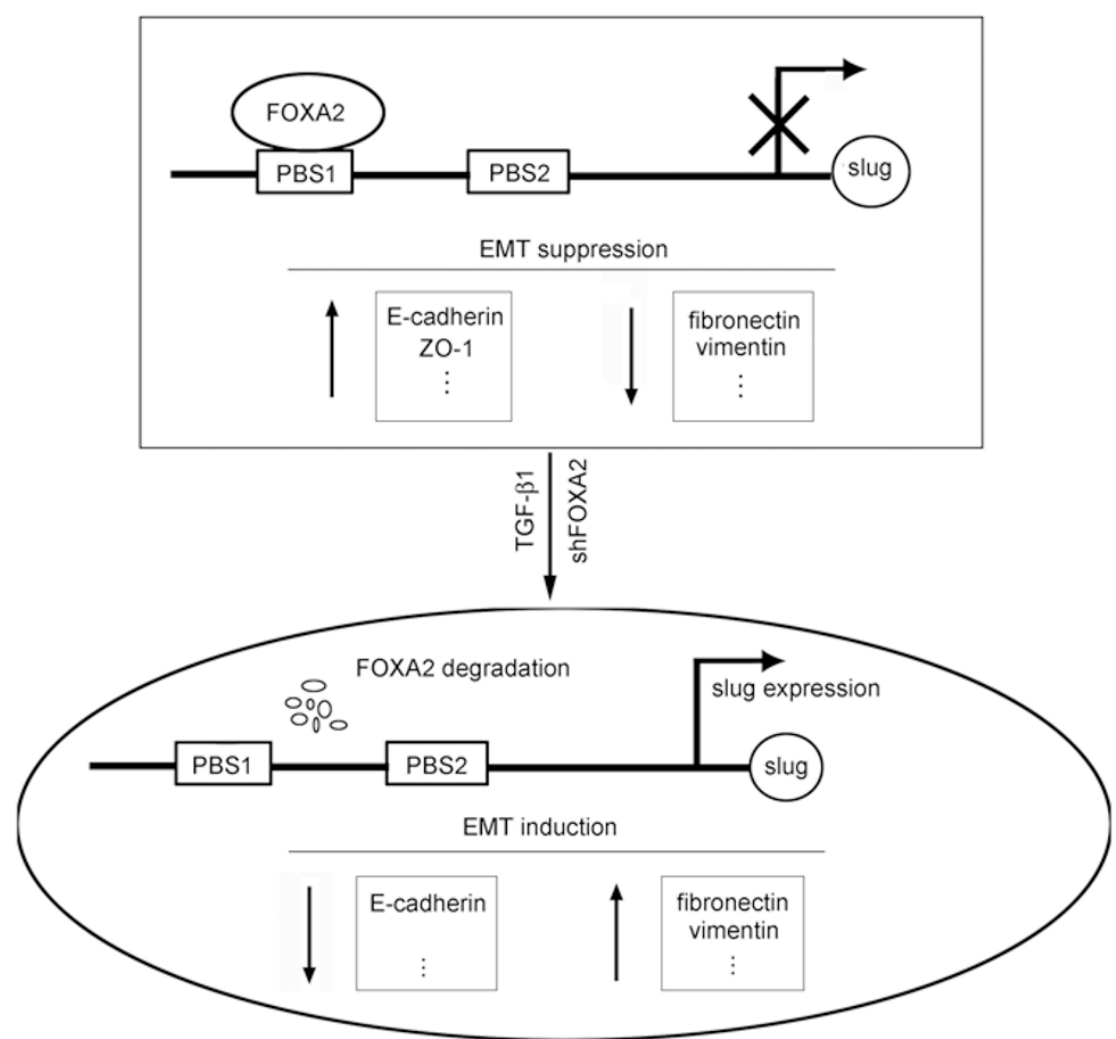

Figure 6 A model for our work. In resting state, FOXA2 suppresses slug transcription. TGF- $\beta 1$ treatment decreases FOXA2 and leads to activation of slug transcription, thus inducing EMT and promoting invasion in human lung cancer cells.

gan genesis [30]. In adulthood, FOXA2 has been shown to control metabolic homeostasis and to contribute to insulin resistance [14].

Very little is known about the roles of FOXA2 in EMT and tumor metastasis. Our study shows that depletion of FOXA2 induces EMT and promotes the invasion of human lung cancer cells into the circulating system. Overexpression of FOXA2 inhibits human lung cancer cell invasion and migration. Because overexpression of FOXA2 inhibited the anchorage-independent cell growth, as shown by decreased colony formation of A549 cells in soft agar (Supplementary information, Figure S3A), whereas knockdown FOXA2 increased the colony formation of cells in soft agar (Supplementary information, Figure S3B), FOXA2 is likely important for anchorage-independent cell survival and growth, which are closely related with cell anoikis. These data suggest that FOXA2 is an important tumor suppressor, which functions in multistages of tumor development. Endogenous FOXA2 protein level was markedly downregulated as early as $3 \mathrm{~h}$ after TGF- $\beta 1$ treatment, but it is not the case for the ectopically expressed flag-tagged FOXA2. The exogenous FOXA2 transcription is controlled by the
H1 promoter, which is not regulated by TGF- $\beta 1$. The fact that TGF- $\beta 1$ treatment did not significantly decrease exogenous FOXA2 indicates that the regulation of FOXA2 level by TGF- $\beta 1$ was not through a posttranscriptional mechanism. In other words, TGF- $\beta 1$ downregulates FOXA2 expression mainly at the transcriptional level. The fact that the levels of FOXA2 mRNA decreased rapidly in response to TGF- $\beta 1$ treatment further supports the above conclusion (Supplementary information, Figure S4A). Nevertheless, how TGF- $\beta 1$ regulates FOXA2 transcription, and whether this is through a Smad-dependent or -independent mechanism remains to be characterized. Interestingly, no FOXA1 expression in A549 cells was observed (Supplementary information, Figure S3A). FOXA1 is weakly expressed in HCC827, but no detectable FOXA1 level was observed in H1299 and H358 (Supplementary information, Figure S4B). The EST profile from NCBI also showed that the expression level of FOXA1 in human lung cancer is much lower than that of FOXA2 (Supplementary information, Figure S4C). Thus, FOXA1 may not fulfill the function of FOXA2 in this case.

As a key mediator of EMT, slug is regulated by TGF$\beta 1$ in a Smad-dependent manner. It has been shown 
that Smad3 forms a complex with myocardin-related transcription factors and binds to the slug promoter to activate slug transcription [31]. However, the mechanism that negatively regulates slug expression remains very poorly understood. In this study, FOXA2 was shown for the first time to suppress slug transcription via a mechanism of binding to the promoter of slug through a conserved binding site. Knockdown of FOXA2 induces slug expression in human lung cancer cells. These findings provide an insight into the complex signaling network involved in slug regulation. In this network, both positive and negative regulators act together to control slug expression. Thus, abnormal changes in either positive or negative signaling molecules can alter slug expression and cause EMT in human lung cancer cells.

Deletion of FOXA2 in the epithelial cells of the developing mouse lung inhibits the differentiation of respiratory epithelial cells and causes neonatal respiratory failure, with the characteristics of respiratory distress syndrome in preterm infants [32]. A recent report showed that FOXA2 plays an important role in epithelialization, and FOXA2-mutant endodermal cells fail to maintain polarity and do not establish proper cellular junctions, and are thus unable to functionally integrate into the endoderm epithelium [33]. Another most recent study showed that FOXA1/2 are negative regulators of EMT in human pancreatic cancer [34]. These results are highly consistent with our findings that FOXA2 is an essential epithelium maintainer in human lung cancer cells. These data suggest that FOXA2 may be a universal regulator of the epithelial and mesenchymal switch in both normal and cancer cells.

In brief summary, we found that FOXA2 is a new TGF- $\beta 1$ target that regulates EMT in human lung cancer cells. FOXA2 inhibits EMT and tumor metastasis by suppressing slug transcription through a conserved binding site in the slug promoter. The DNA-binding region and C-terminal transactivation region II of FOXA2 are required for repressing slug transcription. These results suggest that FOXA2 is a potential new drug target for lung cancer treatment and that it can be used as a prognostic marker for cancer progression.

\section{Materials and Methods}

\section{Cell culture and transfection}

293 T and A549 cells were grown in DMEM containing 10\% FBS supplemented with penicillin $(100 \mathrm{U} / \mathrm{ml})$ and streptomycin (100 mg/ml). H358, H446, and H1299 cells were grown in 1640 medium containing $10 \%$ FBS supplemented with penicillin (100 $\mathrm{U} / \mathrm{ml})$ and streptomycin $(100 \mathrm{mg} / \mathrm{ml})$. AML-12 cells were grown in a 1:1 mixture of DMEM and Ham's F12 medium containing $10 \%$ FBS and supplemented with insulin-transferrin-selenium-X, dexamethasone $(40 \mathrm{ng} / \mathrm{ml})$, penicillin $(100 \mathrm{U} / \mathrm{ml})$, and streptomy- $\operatorname{cin}(100 \mathrm{mg} / \mathrm{ml})$. The cells were incubated at $37{ }^{\circ} \mathrm{C}$ in a humidified atmosphere of $5 \% \mathrm{CO}_{2}$.

\section{Antibodies and reagents}

All cell lines were originally purchased from the American Type Culture Collection (Manassas, VA, USA). Cell culture and transfection reagents were bought from Invitrogen (Carlsbad, CA, USA). TGF- $\beta 1$ was from Chemicon (Millipore, Billerica, MA). Mouse monoclonal antibodies against FOXA2 (ab60721) and flag (F3165.2MG); rabbit polyclonal antibody against slug (ab63119); and mouse monoclonal antibody against $\mathrm{ZO}-1$ and vimentin were purchased from Invitrogen. Mouse monoclonal antibody against E-cadherin was from BD Biosciences (San Jose, CA, USA).

\section{Migration assays}

Cell migration assays were performed using Transwell migration chambers ( $8 \mu \mathrm{m}$ pore size; Costar) according to the vendor's instructions. For A549 cell, 50000 cells were plated into the insert of the well and represent photos were taken under a 200 -fold vision. For other cell lines, 20000 cells were plated into the insert of the well and represent photos were taken under a 100 -fold vision.

Preparation of cell lysates, western blotting, and immunofluorescence staining

Cells were lysed in $10 \mathrm{mM}$ Tris ( $\mathrm{pH}$ 7.4), $1 \mathrm{mM}$ EDTA, 0.5 mM EGTA, $150 \mathrm{mM} \mathrm{NaCl}, 1 \%$ Triton X-100, $50 \mathrm{mM} \mathrm{NaF}, 10 \mathrm{mM}$ $\mathrm{Na}_{4} \mathrm{P}_{2} \mathrm{O}_{7} \cdot 10 \mathrm{H}_{2} \mathrm{O}, 5 \mu \mathrm{g} / \mathrm{ml}$ aprotinin, $5 \mu \mathrm{g} / \mathrm{ml}$ Leupeptin, and $1 \mathrm{mM}$ PMSF. Western blotting and immnofluorescence staining were performed as previously described [35].

\section{${ }^{3} H$-thymidine incorporation analysis}

Cells were labeled with ${ }^{3} \mathrm{H}$-thymidine $(0.1 \mathrm{ACi} / \mathrm{ml})$ in the last 4 $\mathrm{h}$ of incubation. The assay was conducted as previously described [36].

\section{ChIP assay}

ChIP assays were performed according to the manufacturer's instructions (Upstate, 9002). DNA-protein complexes were amplified by PCR for 25 cycles in the presence of $1.5 \mathrm{mM} \mathrm{MgCl}_{2}, 200$ $\mu \mathrm{M}$ dNTPs, and 10 pmol of primers. Precipitated DNA $(10 \%)$ was used for each PCR. PCR products were separated by electrophoresis on $1.5 \%$ low-melt agarose gels and visualized with ethidium bromide. All ChIP experiments were repeated at least three times.

\section{Lentiviral transduction}

Cell lines stably expressing FOXA2 shRNA are established using a vector-based shRNA technique. All shRNAs were from Sigma. The human FOXA2 shRNA target is $5^{\prime}$-ctcctcttaagaagacgac- $3^{\prime}$. The shRNA control (scramble) sequence is $5^{\prime}$-gaattactcctagaaccgc-3'. Lentiviruses were produced by cotransfecting subconfluent 293T (human embryonic kidney) cells with an expression plasmid and a packaging plasmid (pMDLg/pRRE and RSV-Rev) by calcium phosphate transfection. To collect infectious lentiviruses, supernatants were centrifuged to remove cell debris and filtered through $0.45-\mu \mathrm{m}$ filters (Millipore). Cells were infected with the lentivirus containing FOXA2 or scramble nontarget shRNA. The FOXA2 knockdown efficiency was determined by western blotting. To generate cells stably overexpressing FOXA2 or slug, fulllength FOXA2 cDNA or slug cDNA was cloned into a lentiviral vector, and the lentiviruses were prepared as described above and 
transformed into cells.

\section{Blood RNA isolation, reverse transcription and real-time PCR analysis \\ Blood from mice was isolated and red blood cells were lysed.} Total RNA was extracted from A549 cells using Trizol reagent. Then mRNA was reverse transcribed at $42{ }^{\circ} \mathrm{C}$ for $30 \mathrm{~min}$ using ReverTra Ace- $\alpha$ (Toyobo). The presence of circulating tumor cells was assessed as a function of human-specific GAPDH (hGAPDH) expression relative to mouse-specific GAPDH (mGAPDH). Realtime PCR was performed using Power SYBR Green PCR Master Mix with Applied Biosystems 7500 Fast Real-Time PCR system. The relative amount of expression was calculated from a relative standard curve obtained using log dilutions of cDNA containing the gene of interest.

\section{Animal experiments}

Nude mice are used and are bred in SPF animal house. All animal work was done in accordance with a protocol approved by the Shanghai Experimental Animal Center (Chinese Academy of Sciences). Experimental metastasis assay was performed as described by Han et al. [37]. Circulating tumor cells in blood were measured as described by Padua et al. [38]. Control shRNA cells, FOXA2 knockdown cells, control FOXA2 overexpressing cells, and FOXA2 overexpressing cells $\left(1 \times 10^{6}\right.$ cells; $n=3$ mice each $)$ were injected into the lateral tail vein of 5 -week-old nude mice. After 8 weeks, Blood from mice was isolated and red blood cells were lysed. RNA from the remaining cells was extracted for qRTPCR.

\section{Electrophoretic mobility shift assay}

EMSA assays were performed according to the manufacturer's instructions (PIERCE, 20148) with a minor revision. Nondenatured nuclear proteins were extracted and mixed with 5 '-end biotin-labeled probes, and then the mixtures were separated by $6 \%$ nondenatured gel electrophoresis, transferred onto a nylon membrane, and the shift and super-shift bands were examined with ECL using biotin antibody.

\section{Statistical analysis}

Quantitative data are expressed as means \pm SEM. Statistical significance was determined by the Student's $t$-test. A $P$-value of $<0.05$ was considered statistically significant $\left({ }^{*} P<0.05,{ }^{* *} P<\right.$ $0.001)$.

\section{Acknowledgments}

We thank Dr Dangsheng Li (Shanghai Information Center for Life Sciences, China) for critical reading and editing of the paper. We thank members of our laboratory for many helpful discussions. This work was supported by The National Natural Science Foundation of China (30730023, 30721065, 30623003); the National Basic Research Program of China (2007CB947900); and the Science and Technology Committee of Shanghai Municipality (088014199).

\section{Reference}

1 Moustakas A, Heldin CH. Signaling networks guiding epi- thelial-mesenchymal transitions during embryogenesis and cancer progression. Cancer Sci 2007; 98:1512-1520.

2 Tsuji T, Ibaragi S, Hu GF. Epithelial-mesenchymal transition and cell cooperativity in metastasis. Cancer Res 2009; 69:7135-7139.

3 Thiery JP, Acloque H, Huang RY, Nieto MA. Epithelial-mesenchymal transitions in development and disease. Cell 2009; 139:871-890.

4 Cannito S, Novo E, Di Bonzo LV, Busletta C, Colombatto S, Parola M. Epithelial-mesenchymal transition: from molecular mechanisms, redox regulation to implications in human health and disease. Antioxid Redox Signal 2010; 12:1383-1430

5 Thiery JP, Sleeman JP. Complex networks orchestrate epithelial-mesenchymal transitions. Nat Rev Mol Cell Biol 2006; 7:131-142.

6 Huber MA, Kraut N, Beug H. Molecular requirements for epithelial-mesenchymal transition during tumor progression. Curr Opin Cell Biol 2005; 17: 548-558.

7 Heldin CH, Landstrom M, Moustakas A. Mechanism of TGFbeta signaling to growth arrest, apoptosis, and epithelialmesenchymal transition. Curr Opin Cell Biol 2009; 21:166176.

8 Yang Y, Pan X, Lei W, Wang J, Song J. Transforming growth factor-beta1 induces epithelial-to-mesenchymal transition and apoptosis via a cell cycle-dependent mechanism. Oncogene 2006; 25:7235-7244.

9 Katoh M. Human FOX gene family (Review). Int J Oncol 2004; 25:1495-1500.

10 Kaestner KH, Lee KH, Schlondorff J, Hiemisch H, Monaghan AP, Schutz G. Six members of the mouse forkhead gene family are developmentally regulated. Proc Natl Acad Sci USA 1993; 90:7628-7631.

11 Friedman JR, Kaestner KH. The Foxa family of transcription factors in development and metabolism. Cell Mol Life Sci 2006; 63:2317-2328.

12 Bochkis IM, Rubins NE, White P, Furth EE, Friedman JR, Kaestner KH. Hepatocyte-specific ablation of Foxa2 alters bile acid homeostasis and results in endoplasmic reticulum stress. Nat Med 2008; 14:828-836.

13 Wolfrum C, Stoffel M. Coactivation of Foxa2 through Pgc1beta promotes liver fatty acid oxidation and triglyceride/ VLDL secretion. Cell Metab 2006; 3:99-110.

14 Puigserver P, Rodgers JT. Foxa2, a novel transcriptional regulator of insulin sensitivity. Nat Med 2006; 12:38-39.

15 Khoor A, Stahlman MT, Johnson JM, Olson SJ, Whitsett JA. Forkhead box A2 transcription factor is expressed in all types of neuroendocrine lung tumors. Hum Pathol 2004; 35:560564.

16 Lehner F, Kulik U, Klempnauer J, Borlak J. The hepatocyte nuclear factor 6 (HNF6) and FOXA2 are key regulators in colorectal liver metastases. FASEB J 2007; 21:1445-1462.

17 Kasai H, Allen JT, Mason RM, Kamimura T, Zhang Z. TGFbeta1 induces human alveolar epithelial to mesenchymal cell transition (EMT). Respir Res 2005; 6:56.

18 Shi J, Wang DM, Wang CM, et al. Insulin receptor substrate-1 suppresses transforming growth factor-beta1-mediated epithelial-mesenchymal transition. Cancer Res 2009; 69:7180-7187.

19 Thisse C, Thisse B, Postlethwait JH. Expression of snail2, a second member of the zebrafish snail family, in cephalic mesendoderm and presumptive neural crest of wild-type and 
spadetail mutant embryos. Dev Biol 1995; 172:86-99.

20 Hajra KM, Chen DY, Fearon ER. The SLUG zinc-finger protein represses E-cadherin in breast cancer. Cancer Res 2002; 62:1613-1618.

21 Savagner P, Yamada KM, Thiery JP. The zinc-finger protein slug causes desmosome dissociation, an initial and necessary step for growth factor-induced epithelial-mesenchymal transition. J Cell Biol 1997; 137:1403-1419.

22 Medici D, Hay ED, Olsen BR. Snail and slug promote epithelial-mesenchymal transition through beta-catenin-t-cell factor4-dependent expression of transforming growth factor-beta 3. Mol Biol Cell 2008; 19:4875-4887.

23 Shioiri M, Shida T, Koda K, et al. Slug expression is an independent prognostic parameter for poor survival in colorectal carcinoma patients. Br J Cancer 2006; 94:1816-1822.

24 Qian X, Costa RH. Analysis of hepatocyte nuclear factor-3 beta protein domains required for transcriptional activation and nuclear targeting. Nucleic Acids Res 1995; 23:1184-1191.

25 Overdier DG, Porcella A, Costa RH. The DNA-binding specificity of the hepatocyte nuclear factor 3 /forkhead domain is influenced by amino-acid-residues adjacent to the recognition helix. Mol Cell Biol 1994; 14:2755-2766.

26 Pani L, Overdier DG, Porcella A, Qian X, Lai E, Costa RH. Hepatocyte nuclear factor 3 beta contains two transcriptional activation domains, one of which is novel and conserved with the Drosophila fork head protein. Mol Cell Biol 1992; 12:3723-3732.

27 Qian XB, Costa RH. Analysis of hepatocyte nuclear factor3-beta protein domains required for transcriptional activation and nuclear targeting. Nucleic Acids Res 1995; 23:1184-1191.

28 Parkin DM. Global cancer statistics in the year 2000. Lancet Oncol 2001; 2:533-543.

29 Girard L, Zochbauer-Muller S, Virmani AK, Gazdar AF, Minna JD. Genome-wide allelotyping of lung cancer identi- fies new regions of allelic loss, differences between small cell lung cancer and non-small cell lung cancer, and loci clustering. Cancer Res 2000; 60:4894-4906.

30 Sekiya T, Muthurajan UM, Luger K, Tulin AV, Zaret KS. Nucleosome-binding affinity as a primary determinant of the nuclear mobility of the pioneer transcription factor FoxA. Genes Dev 2009; 23:804-809.

31 Morita T, Mayanagi T, Sobue K. Dual roles of myocardinrelated transcription factors in epithelial mesenchymal transition via slug induction and actin remodeling. J Cell Biol 2007; 179:1027-1042.

32 Wan $\mathrm{H}, \mathrm{Xu} \mathrm{Y}$, Ikegami M, et al. Foxa2 is required for transition to air breathing at birth. Proc Natl Acad Sci USA 2004; 101:14449-14454.

33 Burtscher I, Lickert H. Foxa2 regulates polarity and epithelialization in the endoderm germ layer of the mouse embryo. Development 2009; 136:1029-1038.

34 Song Y, Washington MK, Crawford HC. Loss of FOXA1/2 is essential for the epithelial-to-mesenchymal transition in pancreatic cancer. Cancer Res 2010; 70:2115-2125.

35 Zhang KH, Tian HY, Gao X, et al. Ferritin heavy chainmediated iron homeostasis and subsequent increased reactive oxygen species production are essential for epithelial-mesenchymal transition. Cancer Res 2009; 69:5340-5348.

36 Chen HH, Zhao S, Song JG. TGF-beta1 suppresses apoptosis via differential regulation of MAP kinases and ceramide production. Cell Death Differ 2003; 10:516-527.

37 Han HJ, Russo J, Kohwi Y, Kohwi-Shigematsu T. SATB1 reprogrammes gene expression to promote breast tumour growth and metastasis. Nature 2008; 452:187-193.

38 Padua D, Zhang XH, Wang Q, et al. TGFbeta primes breast tumors for lung metastasis seeding through angiopoietin-like 4 . Cell 2008; 133:66-77.

(Supplementary information is linked to the online version of the paper on Cell Research website.) 